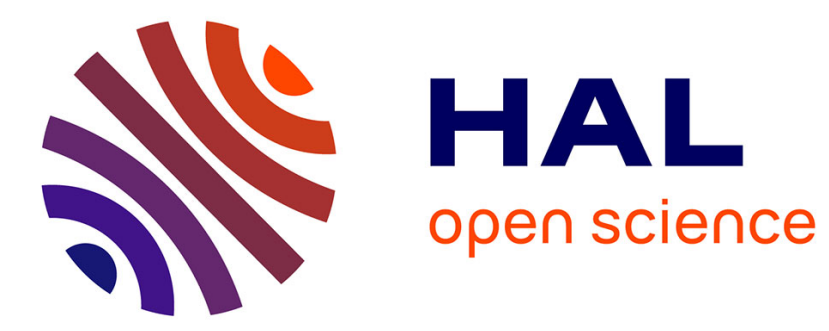

\title{
Semi-analytic inverse method for rubber testing at high strain rates
}

Souheila Aloui, Ramzi Othman, Erwan Verron, Pierrick Guégan

\section{To cite this version:}

Souheila Aloui, Ramzi Othman, Erwan Verron, Pierrick Guégan. Semi-analytic inverse method for rubber testing at high strain rates. Mechanics Research Communications, 2013, 47, pp.97-101. 10.1016/j.mechrescom.2012.10.002 . hal-01007277

\section{HAL Id: hal-01007277 https://hal.science/hal-01007277}

Submitted on 16 Jun 2014

HAL is a multi-disciplinary open access archive for the deposit and dissemination of scientific research documents, whether they are published or not. The documents may come from teaching and research institutions in France or abroad, or from public or private research centers.
L'archive ouverte pluridisciplinaire HAL, est destinée au dépôt et à la diffusion de documents scientifiques de niveau recherche, publiés ou non, émanant des établissements d'enseignement et de recherche français ou étrangers, des laboratoires publics ou privés. 


\title{
Semi-analytic inverse method for rubber testing at high strain rates
}

\author{
Souheila Aloui ${ }^{\mathrm{a}, c, d}$, Ramzi Othman ${ }^{\mathrm{a}, \mathrm{b}}$, Erwan Verron ${ }^{\mathrm{a}}$, Pierrick Guégan ${ }^{\mathrm{a}}$ \\ a LUNAM Université, Institut de Recherche en Génie Civil et Mécanique, UMR CNRS 6183, Ecole Centrale de Nantes, 1 rue de la Noë BP 92101, 44321 Nantes cedex 3, France \\ ${ }^{\mathrm{b}}$ Mechanical Engineering Department, Faculty of Engineering, King Abdulaziz University, P.O. Box 80248, Jeddah 21589, Saudi Arabia \\ ' École d'Architecture et d'Urbanisme de Tunis, Rue de Jérusalem, 2026 Sidi Bou Said, Tunisie \\ ${ }^{\mathrm{d}}$ Applied Mechanics and Systems Research Laboratory, Tunisia Polytechnic School, BP 743, 2048 La Marsa, Tunisia
}

Because of their low mechanical wave speed, high strain rate testing of rubber is highly difficult. Indeed, stress and strain homogeneity is hard to achieve. In this paper, a semi-analytic inverse solution is proposed. This solution is based on a uni-axial stress state assumption in the specimen. Moreover, a new-Hookean law is assumed for rubber. The new method is successfully applied to a high strain rate test on a synthetic rubber.
Keywords:

High strain rate

Soft material

Stress heterogeneity

Elastomer

Hopkinson bar

\section{Introduction}

High strain rate testing of rubber is highly difficult. Indeed, the time needed to achieve stress and strain homogneity is lengthy, because of the low mechanical wave speed of rubbers (Meng and Li, 2003; Song and Chen, 2004; Yang and Shim, 2005; Hong et al., 2008). Precisely, the specimen can be deformed up to some percents of strain before achieving strain and stress homogeneity. In this case, the use of boundary force and displacement measurements to calculate the average stress and strain, respectively, can lead to important errors as it is shown in the section 3.3.

In order to solve this difficulty some works proposed the use of inverse techniques (Gary and Zhao, 1994; Rota, 1997; Zhao, 2003; Kajberg and Lindvist, 2004; Kajberg and Wikman, 2007; Sasso et al., 2008; Sedighi et al., 2010). They assume the knowledge of a constitutive equation which is used to solve a direct problem. The parameters of the constitutive equation are determined by solving and optimisation problem. The inverse techniques are referred as parametric methods. The main advantage of inverse techniques is their stability against noise.

Recently, Aloui et al. (2008) and Othman et al. (2010) proposed a non-parametric method which assumes a uni-axial stress state in the specimen. Moreover, the method necessitates the knowledge of one force at one boundary of the specimen and the whole twodimensional displacement field. Nevertheless, the accuracy of this method is sensitive to the calculation of the acceleration field from the measured displacement field.

By using the same framework as the non-parametric method, the present paper proposes a semi-analytic inverse method to determine rubber behaviour at high strain rates. The proposed new methods sidestep the calculation of the acceleration and uses directly the displacement field. Moreover, it is easy implementable and low-time consuming as compared to inverse techniques based on finite element methods.

\section{Theoretical basics}

We are interested in a prallelipedic or cylindrical specimen of length $L$ as shown in Fig. 1.

For sake of simplicity, the present study is derived for a square cross-section of height and width $h$. Let $\Omega(t)$ be the domain occupied by the specimen at time $t$ during the test and $\Omega_{0}=\Omega(0)$ the domain occupied before the test, i.e., the reference configuration. Each material point of the body is represented by its position in the reference configuration: $\mathbf{X}=X_{1} \mathbf{e}_{1}+X_{2} \mathbf{e}_{2}+X_{3} \mathbf{e}_{3}$. We denote $S_{X_{1}}=\left\{\boldsymbol{\chi} \in \Omega_{0} ; \boldsymbol{\chi} \cdot \mathbf{e}_{1}=X_{1}\right\}$ the cross-section of normal $\mathbf{e}_{1}$ at the abscissa $X_{1}$.

As we consider uniaxial compression experiments, we can assume that cross-sections $S_{X_{1}}$ remain plane during the whole test. In other words, the displacement along $\mathbf{e}_{1}$ of any point in $S_{X_{1}}$ depends only on $X_{1}$ and $t$,

$\mathbf{u}(\mathbf{X}, t) \cdot \mathbf{e}_{1}=u_{1}\left(X_{1}, t\right)$, 


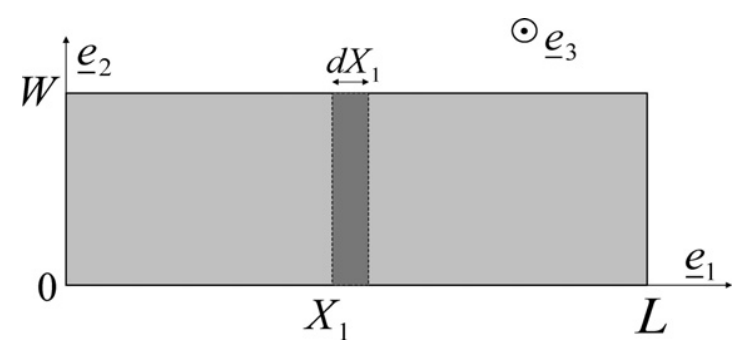

Fig. 1. Schematic of the specimen.

where $\mathbf{u}$ is the displacement field. Similarly, we consider that the force applied to any cross-section $S_{X_{1}}$ is normal to it and depends only on $X_{1}$ and $t$ :

$\mathbf{F}(\mathbf{X}, t)=F_{1}\left(X_{1}, t\right) \mathbf{e}_{1}$.

Applying the second Newton's law, along $\mathbf{e}_{1}$, on a slice of the specimen of length $d X_{1}$, as depicted in Fig. 1, yields to

$\frac{\partial F_{1}}{\partial X_{1}}\left(X_{1}, t\right)=\rho_{0} A_{0} \frac{\partial^{2} u_{1}}{\partial t^{2}}\left(X_{1}, t\right)$.

where $\rho_{0}$ and $A_{0}$ are the specimen initial material density and crosssectional area, respectively. The integration of Eq. 3 with respect to $X_{1}$ leads to the force applied to the cross-section $S_{X_{1}}$ :

$F_{1}\left(X_{1}, t\right)=F_{1}(0, t)+\rho_{0} A_{0} \int_{\xi=0}^{\xi=X_{1}} \frac{\partial^{2} u_{1}}{\partial t^{2}}(\xi, t) d \xi$,

to the unique non-zero component of the first Piola-Kirchhoff stress tensor, i.e. the axial nominal stress,

$P_{11}\left(X_{1}, t\right)=\frac{F_{1}\left(X_{1}, t\right)}{A_{0}}=\frac{F_{1}(0, t)}{A_{0}}+\rho_{0} \int_{\xi=0}^{\xi=X_{1}} \frac{\partial^{2} u_{1}}{\partial t^{2}}(\xi, t) d \xi$,

and to the unique non-zero component of the Cauchy stress tensor, i.e. the axial true stress

$\sigma_{11}\left(X_{1}, t\right)=\frac{F_{1}\left(X_{1}, t\right)}{A\left(X_{1}, t\right)}=\frac{F_{1}(0, t)}{A\left(X_{1}, t\right)}+\frac{\rho_{0} A_{0}}{A\left(X_{1}, t\right)} \int_{\xi=0}^{\xi=X_{1}} \frac{\partial^{2} u_{1}}{\partial t^{2}}(\xi, t) d \xi$,

in which $A\left(X_{1}, t\right)$ is the area of the cross-section $S_{X_{1}}$ at time $t$.

In the non-parametric method proposed in Refs. (Aloui et al., 2008; Othman et al., 2010), Eqs. (5) and (6) are used to determine the stress field from measurements of the force at one boundary of the specimen, and of the displacement field. However, the displacement field must be differentiated twice to get the acceleration field and it may amplify experimental noise.

In the following, the material is considered homogeneous and we further assume the knowledge of its constitutive equation that can be written as

$P_{11}\left(X_{1}, t\right)=\mathcal{B}_{c_{1}, c_{2}, \ldots, c_{n}}\left(\mathbf{E}\left(X_{1}, t\right)\right)$

where $\mathcal{B}$ is a map, $c_{1}, c_{2}, \ldots, c_{n}$ are the material parameters and $\mathbf{E}\left(X_{1}, t\right)$ is the Green-Lagrange strain tensor defined as

$\mathbf{E}=\frac{1}{2}\left(\frac{\partial \mathbf{u}^{T}}{\partial \mathbf{X}}+\frac{\partial \mathbf{u}}{\partial \mathbf{X}}\right)+\frac{1}{2} \frac{\partial \mathbf{u}^{T}}{\partial \mathbf{X}} \frac{\partial \mathbf{u}}{\partial \mathbf{X}}$.

Similarly, the Cauchy stress can be written as

$\sigma_{11}\left(X_{1}, t\right)=\beta_{c_{1}, c_{2}, \ldots, c_{n}}\left(\boldsymbol{\varepsilon}\left(X_{1}, t\right)\right)$.

In this last equation, $\beta$ holds for a map and $\varepsilon$ is the logarithmic or Hencky strain tensor defined by

$\boldsymbol{\varepsilon}=\frac{1}{2} \ln (\mathbf{I}+2 \mathbf{E})$

in which $\mathbf{I}$ is the $3 \times 3$ identity tensor.
Eqs. (5) and (7) are two different expressions of the uniaxial nominal stress. The former equation gives the nominal stress in terms of both a force at one specimen boundary and the displacement field, and the latter one expresses the nominal stress in terms of the Green-Lagrange strain tensor and material parameters. By matching these two expressions of the nominal stress, we can determine the materials constants. It is to note that a similar approach can be adopted with the true stress, i.e. considering Eqs.(6) and (9). As we are interested in applying our method to large strain of rubber-like materials for which the nominal stress is the most relevant stress measure, only Eqs. (5) and (7) will be considered. In order to avoid the double differentiation of the displacement field with respect to time in Eq.(5), the following integrated quantity is defined

$$
\begin{aligned}
\Pi_{11}\left(X_{1}, t\right) & \equiv \int_{0}^{t} \int_{0}^{\tau} P_{11}\left(X_{1}, \tau^{\prime}\right) d \tau^{\prime} d \tau \\
& =\int_{0}^{t} \int_{0}^{\tau} P_{11}\left(0, \tau^{\prime}\right) d \tau^{\prime} d \tau+\rho_{0} \int_{\xi=0}^{\xi=X_{1}} u_{1}(\xi, t) d \xi .
\end{aligned}
$$

Its expression issued from Eq. (7) is

$\Pi_{11}\left(X_{1}, t\right)=\int_{0}^{t} \int_{0}^{\tau} \mathcal{B}_{c_{1}, c_{2}, \ldots, c_{n}}\left(\mathbf{E}\left(X_{1}, \tau^{\prime}\right)\right) d \tau^{\prime} d \tau$.

Matching the expressions of $\Pi_{11}$ of Eqs. (11) and (12) to determine the set of material parameters $\left\{c_{1}, c_{2}, \ldots, c_{n}\right\}$ can be achieved by minimizing the cumulated (in both time and space) difference between these two quantities, i.e. the objective function $\mathcal{F}$ that depends on the material parameters,

$$
\begin{aligned}
\mathcal{F}\left(c_{1}, c_{2}, \ldots, c_{n}\right)= & \int_{0}^{L} \int_{0}^{T}\left(\int_{0}^{t} \int_{0}^{\tau} \mathcal{B}_{c_{1}, c_{2}, \ldots, c_{n}}\left(\mathbf{E}\left(X_{1}, \tau^{\prime}\right)\right) d \tau^{\prime} d \tau\right. \\
& \left.-\int_{0}^{t} \int_{0}^{\tau} P_{11}\left(0, \tau^{\prime}\right) d \tau^{\prime} d \tau-\rho_{0} \int_{0}^{X_{1}} u_{1}(\xi, t) d \xi\right)^{2} d t d X_{1}
\end{aligned}
$$

where $T$ is the total test duration.

By minimizing the objective function $\mathcal{F}$ with respect to the material parameters $c_{1}, c_{2}, \ldots, c_{n}$, we obtain the constitutive Eq. (7). This method is referred to as the semi-analytical inverse method. Indeed, the objective function is defined analytically; integrals are computed numerically and in some special cases the minimization problem can be solved analytically.

\section{Application to rubberlike materials}

In this section, we apply the semi-analytical method to a synthetic elastomer (Criblex 80). The density of this material is equal to $1272 \mathrm{~kg} / \mathrm{m}^{3}$. We consider cubic samples $(L=h=10 \mathrm{~mm})$. The specimens are painted by a speckle to apply digital image correlation (DIC). Note $S_{u}=\left\{\mathbf{X} \in \Omega_{0} ; X_{2}=\mathbf{X} . \mathbf{e}_{2}=h\right\}$ be the painted (filmed) surface as shown in Fig. 1.

\subsection{Experimental set-up}

The experiments are conducted with a direct impact Hopkinson bar (Klepaczko, 1994). In our set-up, the input bar is removed; then the sample is directly impacted by the projectile. As suggested in (Chen et al., 1999), we used a hollow aluminum bar (inner diameter $14 \mathrm{~mm}$ and outer diameter $16 \mathrm{~mm}$ ) to increase signal-to-noise ratio. This bar works as an output bar and is used to measure the output force $\hat{F}_{\text {out }}(t)$; it is then instrumented with a strain gauge station to measure the transmitted strain wave $\varepsilon_{\text {tra }}(t)$. The output force reads:

$\hat{F}_{\text {out }}(t)=E_{b} A_{b} \hat{\varepsilon}_{0, \text { tra }}(t)$,

where $E_{b}$ and $A_{b}$ are the bar Young modulus and cross-sectional area, respectively, and $\hat{\varepsilon}_{0 \text {,tra }}$ stands for the transmitted wave at 
the bar/specimen interface, calculated by shifting the transmitted wave $\hat{\epsilon}_{\text {tra }}$ measured at the gauge station. More precisely, the Fourier components of these two waves, $\varepsilon_{\text {tra }}$ and $\varepsilon_{0, \text { tra }}$, are related by the following equation:

$\tilde{\hat{\varepsilon}}_{0, \operatorname{tra}}(\omega)=e^{i \xi(\omega) a} \tilde{\hat{\varepsilon}}_{\operatorname{tra}}(\omega)$,

where $a$ is the distance between the gauge station and the barspecimen interface, $i^{2}=-1, \xi(\omega)$ is the dispersion relation and the notation $\sim$ stands for the Fourier transform. Here, the dispersion relation is determined by the bar resonances technique as proposed in (Othman et al., 2002). Moreover, we use a 5-mm thick steel disc as a grip both to support the specimen and to apply a uniform force on the cross-section of the hollow bar; this grip does not alter wave propagation because its thickness is very small as compared to wavelengths. Considering the bar-specimen interface as the origin of the $\mathbf{e}_{1}$-axis, the force at this point is equal to the output force, i.e. $\hat{F}_{0}(t)=\hat{F}_{\text {out }}(t)$. Finally, the displacement and strain fields are calculated by applying the DIC technique to images obtained with the high speed PHOTRON Ultima APX video camera; the acquiring frequency is 100,000 frames/s and the resolution is the same as used for the intermediate strain rate tests, i.e. $128 \times 248 \mathrm{pix}^{2}$.

\subsection{Analysis}

In this section, we detail how the equations of Section 2 are applied to analyze our experimental data. Recalling the assumption that the displacement $u_{1}$ only depends on the $X_{1}$-coordinate, this displacement in $S_{u}$ is averaged over the $\mathbf{e}_{3}$-direction:

$\hat{u}_{1}\left(X_{1}, t\right)=\frac{1}{h} \int_{0}^{h} \hat{u}_{1}\left(X_{1}, X_{2}=h, X_{3}, t\right) d X_{3}$.

and similarly, the uniaxial Hencky strain component is

$\hat{\varepsilon}_{11}\left(X_{1}, t\right)=\frac{1}{h} \int_{0}^{h} \hat{\varepsilon}_{11}\left(X_{1}, X_{2}=h, X_{3}, t\right) d X_{3}$.

Then, considering that the $\mathbf{e}_{1}$-direction is a principal strain direction in both the reference and the deformed configuration (because the test is uniaxial) and recalling Eq. (8), the estimation of nominal strain is deduced as follows

$\hat{E}_{11}\left(X_{1}, t\right)=\frac{1}{2}\left[\exp \left(2 \hat{\varepsilon}_{11}\left(X_{1}, t\right)\right)-1\right]$.

and the estimation of the corresponding stretch ratio $\lambda_{1}$ is

$\hat{\lambda}_{1}\left(X_{1}, t\right)=\exp \left(\hat{\varepsilon}_{11}\left(X_{1}, t\right)\right)$.

Eq. (11) gives a first way to estimate $\Pi_{11}\left(X_{1}, t\right)$,

$\hat{\Pi}_{11}^{(1)}\left(X_{1}, t\right)=\int_{0}^{t} \int_{0}^{\tau} \frac{\hat{F}_{0}\left(\tau^{\prime}\right)}{A_{0}} d \tau^{\prime} d \tau+\rho_{0} \int_{\xi=0}^{\xi=X_{1}} \hat{u}_{1}(\xi, t) d \xi$.

Then, to define the second estimation of $\Pi_{11}\left(X_{1}, t\right)$ given by Eq. (7), we must consider a constitutive equation. Following the classical assumptions (see for example (Treloar, 1975)), the elastomer is assumed homogeneous, isotropic, incompressible and hyperelastic, i.e. its mechanical response is elastic and can be described by a strain energy density; moreover as the strain remain moderate, we consider the neo-Hookean model which involves only one material parameter and is quiet sufficient for moderate strain (Marckmann and Verron, 2006). Thus, the nominal stress is

$P_{11}\left(X_{1}, t\right)=2 C\left(\lambda_{1}\left(X_{1}, t\right)-\frac{1}{\left[\lambda_{1}\left(X_{1}, t\right)\right]^{2}}\right)$

where $C$ is the unique material parameter. Thus,

$\hat{\Pi}_{11}^{(2)}\left(X_{1}, t\right)=2 C \int_{0}^{t} \int_{0}^{\tau}\left(\hat{\lambda}_{1}\left(X_{1}, \tau^{\prime}\right)+\frac{1}{\left[\hat{\lambda}_{1}\left(X_{1}, \tau^{\prime}\right)\right]^{2}}\right) d \tau^{\prime} d \tau \equiv C \hat{\Phi}\left(X_{1}, t\right)$.

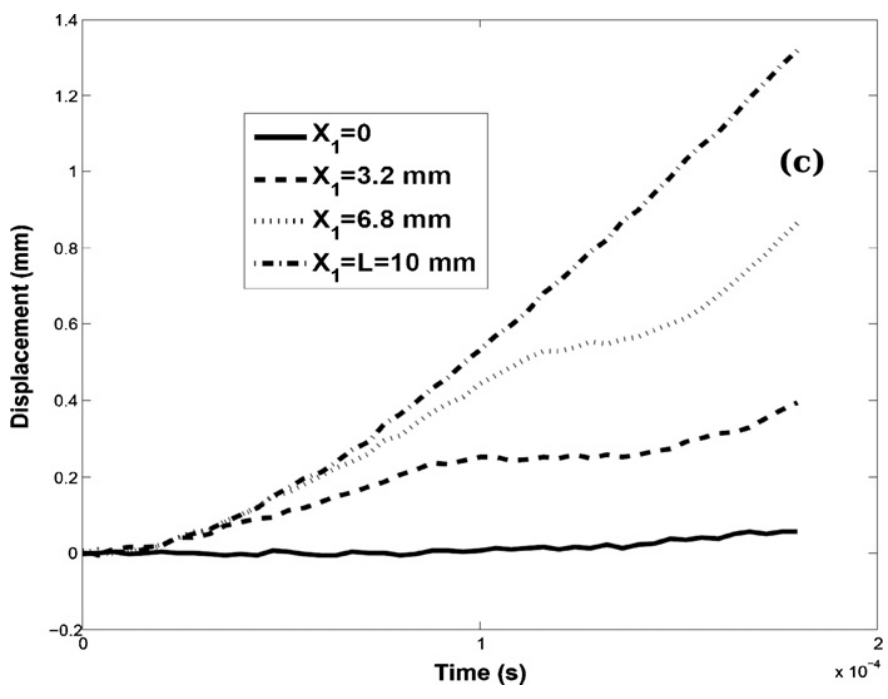

Fig. 2. Displacement $\hat{u}_{1}$ in four points measured by DIC technique.

So, we have two estimations of $\Pi_{11}\left(X_{1}, t\right)$ which are entirely determined by experimental data except the material parameter $C$. This parameter can then be obtained by the least square method applied to the function

$\hat{\mathcal{F}}(C)=\int_{0}^{L} \int_{0}^{T}\left(\hat{\Pi}_{11}^{(1)}\left(X_{1}, t\right)-C \hat{\Phi}\left(X_{1}, t\right)\right)^{2} d t d X_{1}$.

In this simple case, it is obvious that the minimum of $\hat{\mathcal{F}}$ is achieved for

$\hat{C}=\frac{\int_{0}^{L} \int_{0}^{T} \hat{\Pi}_{11}^{(1)}\left(X_{1}, t\right) \hat{\Phi}\left(X_{1}, t\right) d t d X_{1}}{\int_{0}^{L} \int_{0}^{T}\left(\hat{\Phi}\left(X_{1}, t\right)\right)^{2} d t d X_{1}}$.

Moreover, the error of the method is the error committed on the value of $\Pi_{11}\left(X_{1}, t\right)$ which can be defined as the value of the error function at $\hat{C}$, i.e. $\mathcal{F}(C=\hat{C})$, as compared to the mean value of the two estimations of $\Pi_{11}$ :

$\mathcal{X} \equiv 2 \frac{\int_{0}^{L} \int_{0}^{T}\left(\hat{\Pi}_{11}^{(1)}\left(X_{1}, t\right)-\hat{C} \hat{\Phi}\left(X_{1}, t\right)\right)^{2} d t d X_{1}}{\int_{0}^{L} \int_{0}^{T}\left(\hat{\Pi}_{11}^{(1)}\left(X_{1}, t\right)+\hat{C} \hat{\Phi}\left(X_{1}, t\right)\right)^{2} d t d X_{1}}$.

\subsection{Results}

Fields of axial displacement $\hat{u}_{1}$ and first diagonal component of the strain tensor $\hat{\varepsilon}_{11}$ are obtained by DIC technique; they are depicted in Figs. 2 and 3, respectively. These data are analyzed by the semi-analytical inverse method presented above. We then obtain the estimation $\hat{C}$ of the material constant $C$. Moreover, we calculate the error defined in Eq. (25). The values of $\hat{C}$ and $\mathcal{X}$ are given in Table 1.

Knowing $\hat{C}$ and $\hat{\varepsilon}_{11}$, the Cauchy stress field is calculated as

$\hat{\sigma}_{11}=\hat{C}\left[\exp \left(2 \hat{\varepsilon}_{11}\right)-\exp \left(-\hat{\varepsilon}_{11}\right)\right]$.

In order to validate the semi-analytic inverse method, it is compared to three other methods:

Table 1

Material constant $\hat{C}$ and error of the method $\mathcal{X}$.

\begin{tabular}{ll}
\hline & High strain rate \\
\hline$\hat{C}(\mathrm{MPa})$ & 7.83 \\
$\mathcal{X}(\%)$ & 4.54 \\
\hline
\end{tabular}




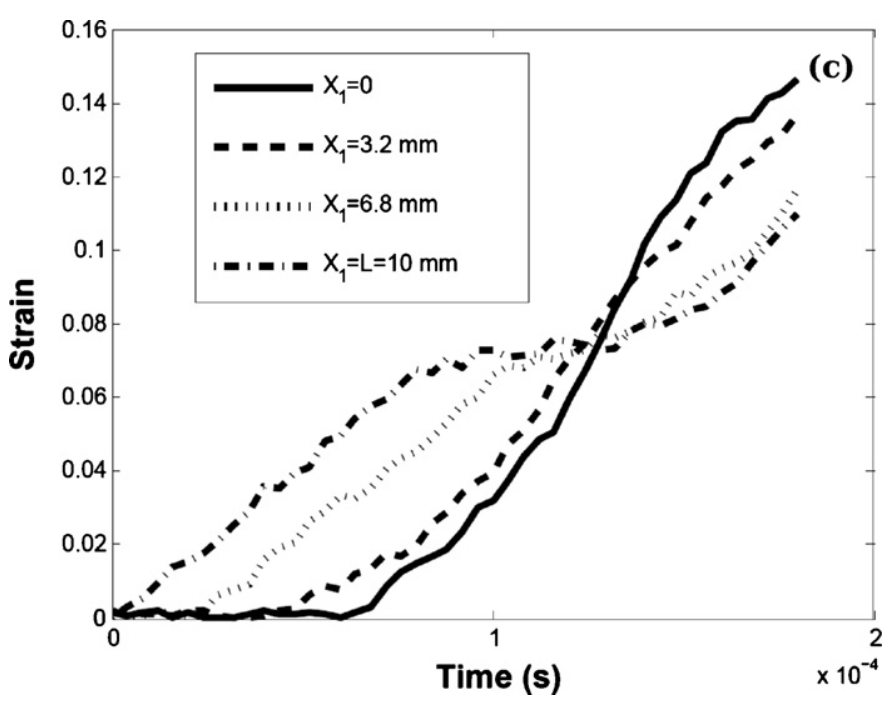

Fig. 3. Strain $\hat{\varepsilon}_{11}$ in four points measured by DIC technique.

- the conventional method which assumes the homogeneity of strain and stress fields; average strain and stress are simply determined by the measurement of the force and the displacement at one of the boundaries,

- the non-parametric method developed in (Aloui et al., 2008; Othman et al., 2010),

- and the complete numerical inverse method that consists in solving numerically the direct and optimization problems simultaneously in (Kajberg and Lindvist, 2004; Kajberg and Wikman, 2007). Here, the direct problem is solved by Abaqus and the optimization problem by Matlab.

Fig. 4 depicts the Cauchy stress-nominal strain curves obtained by the four methods for three different strain rates.

The results present important discrepancies. The curve obtained with the conventional method is obviously not adapted because it assumes the homogeneity of the stress and strain fields: according to this curve, the material is deformed up to $5 \%$ with almost no force applied. Nevertheless, it is not an easy task to determine which of the three other methods gives the best result. The non-parametric method gives the lowest stress curve. This can be explained in two ways. Firstly, this method involves numerical filters for the

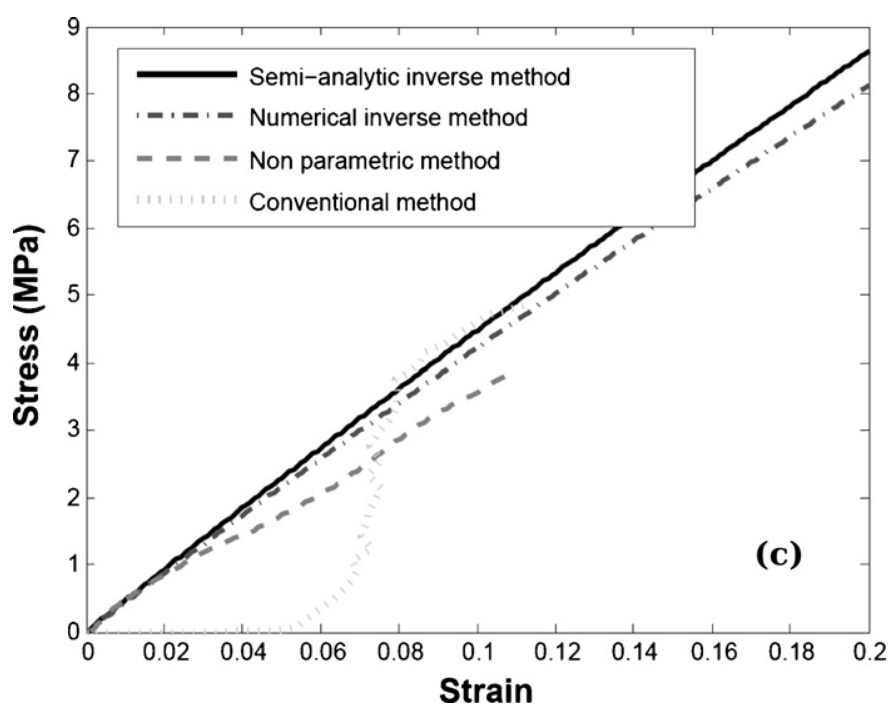

Fig. 4. Comparison of stress $\left(\hat{\sigma}_{11}\right)$-strain $\left(\hat{\varepsilon}_{11}\right)$ curves obtained by four methods. measurement of the displacement field, which surely filters inertia effects. Therefore, the non-parametric method would only partially correct the heterogeneity of the stress field; and then would underestimate it. Secondly, the two inverse methods,i.e. the present one and the numerical inverse method, assume a neo-Hookean constitutive equation for the material; this choice can lead to some discrepancy if this constitutive equation is not well-adapted to the material. Finally, the semi-analytic and the non-parametric methods are simple to implement and are highly less time consuming than the complete numerical inverse method because they do not involve finite element computations.

\section{Conclusion}

In this work, we are interested in the determination of constitutive equation for soft materials using uniaxial dynamic heterogeneous experiments. This paper has proposed a semi-analytic inverse method for the identification of the stress field. It is an alternative solution to the complete numerical inverse method (Kajberg and Lindvist, 2004; Kajberg and Wikman, 2007) and the non-parametric method (Aloui et al., 2008; Othman et al., 2010). It is based on two main assumptions: (i) the cross-sections remain plane during the test and (ii) the force applied to any cross-section is normal to it. Obviously, these assumptions are valid for uniaxial experiments. Compared to the complete numerical inverse method, the semi-analytic inverse method is simpler and highly less time consuming. Compared to the non-parametric method, the semi-analytic method do not need the double numerical differentiation of the displacement field.

The semi-analytic inverse method, presented in this paper, necessitates the a priori choice of the constitutive equation, contrarily to the non-parametric method. Fortunately, Eq. (21) can be rewritten easily to take into account several material coefficients, thus different hyperelastic models (Hartmann, 2001). The only difference will be that, instead of solving a one-dimensional minimization, one will have a multiparametric objective function. In this work, only the neo-Hookean law was considered. Indeed, fitting multiple material parameters with only one uniaxial experiment will lead to an ill-conditioned identification problem (Ogden et al., 3). Therefore, adding more material parameters, thus more refined constitutive equation, is, perhaps, not necessary. In line with this, visco-elastic effects are ignored here, however important. Indeed, it is hard to reveal the visco-elastic behaviour of the rubber with only one almost-constant-strain-rate monotonous uniaxial experiment.

Even if we argue that it is not easy to make a straightforward choice between the three different methods, we suggest the use of both the non-parametric and the semi-analytic inverse methods: both are simple to apply and are not time consuming. Thus, one can try to improve the results given by the two methods by (i) carefully adjusting the filters in the non-parametric method, and (ii) considering different hyperelastic models for the semi-analytic method. Following these advices will conduct to the best results one can afford for heterogeneous dynamic experiments.

\section{References}

Aloui, S., Othman, R., Poitou, A., Guégan, P., El-Borgi, S., 2008. Non-parametric identification of the non-homogeneous stress in high strain-rate uni-axial experiments. Mechanics Research Communications 35, 392-397.

Chen, W., Zhang, B., Forrestal, M.J., 1999. A split Hopkinson bar technique for lowimpedance materials. Experimental Mechanics 39, 80-85.

Gary, G., Zhao, H., 1994. Dépouillement de l'essai aux barres de Hopkinson par une technique de calcul inverse. Journal de Physique IV C8, 89-94.

Hartmann, S., 2001. Numerical studies on the identification of the material parameters of Rivlin's hyperelasticity using tension-torsion tests. Acta Mechanica 148 129-155. 
Hong, L, Li, X., Liu, X., Zhou, Z., Ye, Z., Yin, T., 2008. Stress uniformity process of specimens in SHPB test under different loading conditions of rectangular and half-sine input waves. Transactions of Tianjin University 14, 450-456.

Kajberg, J., Lindvist, G., 2004. Characterisation of materials subjected to large strain rates by inverse modelling based on in-plane displacement fields. International Journal of Solids and Structures 41, 3439-3459.

Kajberg, J., Wikman, B., 2007. Viscoplastic parameter estimation by high strainrate experiments and inverse modelling - speckle measurements and high-speed photography. International Journal of Solids and Structures 44, $145-164$.

Klepaczko, J.R., 1994. An experimental technique for shear testing at high and very high strain rates - the case of mild-steel. International Journal of Impact Engineering 15, 25-39.

Marckmann, G., Verron, E., 2006. Comparison of hyperelastic models for rubberlike materials. Rubber Chemistry and Technology 79, 835858.

Meng, H., Li, Q.M., 2003. Correlation between the accuracy of a SHPB test and the stress uniformity based on numerical experiments. International Journal of Impact Engineering 28, 537-555.

Ogden, R.W., Saccomandi, G., Sgura, I., 2004. Fitting hyperelastic models to experimental data. Computational Mechanics 3, 484.
Othman, R., Blanc, R.H., Bussac, M.N., Collet, P., Gary, G., 2002. Identification of the dispersion relation in rods - identification de la relation de dispersion dans les barres. Comptes rendus Mecanique 320, 849-855.

Othman, R., Aloui, S., Poitou, A., 2010. Identification of non-homogeneous stress fields in dynamic experiments with a non-parametric method. Polymer Testing 29, 616-623.

Rota, L., 1997. Application des méthodes inverses au dépouillement de l'essai aux barres de Hopkinson, Ph.D. Thesis, Ecole Polytechnique, France.

Sasso, M., Newaz, G., Amodio, D., 2008. Material characterization at high strain rate by Hopkinson bar tests and finite element optimization. Materials Science and Engineering A 487, 289-300.

Sedighi, M., Khandaei, M., Shokrollahi, H., 2010. An approach in parametric identification of high strain rate constitutive model using Hopkinson pressure bar test results. Materials Science and Engineering A 527, 3521-3528.

Song, B., Chen, W., 2004. Dynamic stress equilibration in split Hopkinson pressure bar tests on soft materials. Experimental Mechanics 44, 300-312.

Treloar, L.R.G., 1975. The Physics of Rubber Elasticity. Oxford University Press, Oxford.

Yang, L.M., Shim, V.P.W., 2005. An analysis of stress uniformity in split Hopkinson bar test specimens. International Journal of Impact Engineering 31, 129-150.

Zhao, H., 2003. Material behaviour characterisation using SHPB techniques, tests and simulations. Computers and Structures 81, 1301-1310. 